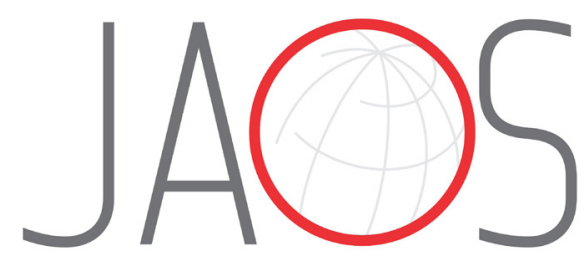

JOURNAL OF APPLIED ORAL SCIENCE

\section{Clinical and microbiological evaluation of non-surgical periodontal therapy in obese and non-obese individuals with periodontitis: a 9-month prospective longitudinal study}

\section{Abstract}

Felipe da Silva PERALTA ${ }^{1}$

Sheila Cavalca CORTELLI ${ }^{1}$ i

Emanuel Silva ROVAI ${ }^{1}$

Davi Romeiro AQUINO ${ }^{1}$

Taís Browne MIRANDA ${ }^{1}$

Fernando Oliveira COSTA ${ }^{2}$ José Roberto CORTELLI ${ }^{1}$ iD
Objective: Obesity is a chronic disease that negatively affects an individual's general and oral health. The present study aimed to compare the clinical and microbiological effects of non-surgical periodontal therapy with the full mouth disinfection (FMD) protocol on obese and non-obese individuals at 9 months post-therapy. Methodology: This clinical study was first submitted and approved by the Ethics Committee. Fifty-five obese patients and 39 nonobese patients with periodontitis were evaluated. The full-mouth periodontal clinical parameters, clinical attachment level $(C A L)$, probing depth (PD), gingival index (GI), and plaque index (PI), were monitored at baseline, 3, 6, and 9 months after periodontal treatment with full mouth disinfection (FMD) protocol. The mean count of Tannerella forsythia, Porphyromonas gingivalis, Treponema Denticola, and Aggregatibacter actinomycetemcomitans was determined by quantitative polymerase chain reaction on subgingival biofilm samples. Demographic data were assessed by Chi-square test. For clinical and microbiological parameters, two-factor repeated-measures ANOVA was used. Results: In both groups, periodontal therapy using the one-stage full-mouth disinfection protocol significantly improved CAL, PD, GI, and PI $(p<0.05)$. Obese and non-obese patients equally responded to non-surgical periodontal therapy $(p>0.05)$. Microbial count found no major differences $(p>0.05)$ between obese and non-obese individuals who had undergone non-surgical periodontal therapy. Conclusions: Obesity did not affect the clinical and microbiological outcomes of non-surgical periodontal therapy.

Keywords: Obesity. Periodontitis. Body mass index. Periodontal disease.
Submitted: November 20, 2019 Modification: March 18, 2020 Accepted: March 23, 2020
'Universidade de Taubaté, Departamento de Odontologia, Taubaté, São Paulo, Brasil. ${ }^{2}$ Universidade Federal de Minas Gerais, Faculdade de Odontologia, Departamento de Periodontia, Belo Horizonte, Minhas Gerais, Brasil. 


\section{Introduction}

Periodontitis is a highly prevalent chronic inflammatory disease characterized by the progressive destruction of the tooth-supporting tissues, resulting in tooth loss. ${ }^{1,2}$ Periodontitis represents not only a simple bacterial infection, but also a complex interaction among host inflammatory responses, subgingival biofilm, and host modifying factors. ${ }^{3}$

Among host modifying factors, several systemic diseases have been associated with periodontitis, including obesity. ${ }^{4,5}$ Obesity is a chronic disease that affects the individual's general and oral health. ${ }^{6,7}$ The cellular and molecular mechanisms that might explain obesity and periodontitis relationship are systemic inflammatory changes in tumor necrosis factor (TNF)-a, Interleukin-6 (IL-6), oxidative stress, and adiponectin and leptin levels, which may result in greater susceptibility to chronic inflammatory diseases and infections. ${ }^{8-10}$

Several epidemiological studies have established the relationship between obesity and periodontal disease. ${ }^{11-13}$ Cross-sectional studies have demonstrated that obese individuals present greater odds-ratio for periodontitis than non-obese. ${ }^{11,13}$ Moreover, longitudinal studies demonstrated that obesity is associated with increased periodontal attachment loss and inflammation, ${ }^{12,14,15}$ and systematic reviews showed that periodontitis severity and extension are associated with increased levels of overweight. 16-18

Previous reports showed that obese individuals present impaired wound healing and more infectious complications. ${ }^{19,20}$ Therefore, the question of whether these individuals would have a worse response to periodontal therapy than non-obese individuals was posed, and a few clinical studies were conducted. ${ }^{20-25}$ Nonetheless, a systematic review evaluating obesity on periodontal therapy showed that the low quality of evidence is mainly due to the lack of studies, shortterm follow-ups, and inconsistent data, indicating the need for further studies. ${ }^{26,27}$

As inflammatory changes in obese individuals may impair wound healing and worsen periodontal status, this study hypothesized that obese individuals would present a worse response to periodontal therapy than non-obese ones. Although there are other studies on this topic, this is the first to verify the effectiveness of the chemical-mechanical therapy with the FMD protocol in obese individuals.
Thus, the aim of this prospective 9-month clinical study was to compare the clinical and microbiological effects of non-surgical periodontal therapy with the FMD protocol on obese and non-obese individuals.

\section{Methodology}

This 9-month, parallel group, single-center, clinical study was registered at Clinicaltrials.gov (NCT03103204) and approved by the Ethics Committee (protocol No. 36828114.4.0000.5501). Participants provided prior written informed consent for enrolling in this clinical study, composed of baseline, 3, 6 and 9 month post-therapy follow ups.

\section{Participants}

The study population was composed of participants with periodontitis referred to the Dental Specialties Center of Joinville-SC, in 2017. A total of 94 individuals of both genders, 45 years old or older, and presenting at least 12 natural teeth, body mass index (BMI) > $18.5 \mathrm{~kg} / \mathrm{m}^{2}$ and periodontitis were recruited. This prospective study included participants with moderate, severe, and advanced periodontitis (stage II: established periodontitis with characteristic damages caused to tooth support, including interdental CAL from 3 to $4 \mathrm{~mm}$, maximum PPD $\leq 5 \mathrm{~mm}$, and radiographic bone loss at coronal third between $15 \%$ to $33 \%$; stage III and IV: - at least interdental CAL $\geq 5 \mathrm{~mm}$, PPD $\geq 6$ $\mathrm{mm}$ and radiographic bone loss extending to mild-third of the root), as described by Tonneti, et al. ${ }^{28}$ (2018).

Sample size was calculated from the primary outcome data of previous studies, based on the clinical attachment level (CAL) gain and probing depth (PD) reduction. ${ }^{29,30}$ Microbial count reduction and the association between clinical and microbiological reductions were chosen as secondary outcome, based on the posteriori mean microbial counts observed in this study. Assuming a $5 \% \alpha$, a $90 \%$ power, and a $15 \%$ minimum PD reduction difference between groups, a total of 28 individuals within each group would be necessary. Notably, in this study the coefficient of variation for microbial count was $\sim 15 \%$, indicating the study outcome precision. ${ }^{29}$ Considering a dropout of up to $\sim 20 \%$, a total of 34 participants in each group were initially regarded as appropriate.

Exclusion criteria were orthodontic devices, pregnancy or breast-feeding, systemic diseases or 
other conditions that could influence the periodontal status (other than diabetes), alcohol abuse, medical condition requiring prophylactic antibiotic coverage, use of systemic antibiotics and/or anti-inflammatory drugs six months prior to the study, and history of periodontal therapy within six months prior to the study.

\section{Anthropometric measurements}

Anthropometric measurements (weight, height, and waist circumference) were recorded at both baseline and 9 months. BMI was calculated as an indicator of total adiposity regarding obesity, by dividing bodyweight (kilograms) by the square of body height (meters). Obesity was defined as BMI $\geq 30 \mathrm{~kg} /$ $\mathrm{m}^{2} .{ }^{31}$ Alongside BMI, the following waist circumference reference-values were considered as indicators of obesity: > $102 \mathrm{~cm}$ for men and $>88 \mathrm{~cm}$ for women. ${ }^{31}$

Individuals selected were divided into two groups, according to their body mass index (BMI) and waist circumference.

Non-obese group $(n=39)$, BMI $\leq 29.9 \mathrm{~kg} / \mathrm{m}^{2}$ and waist circumference $<102 \mathrm{~cm}$ for men and $<88 \mathrm{~cm}$ for women.

Obese group ( $n=55)$, BMI $\geq 30 \mathrm{~kg} / \mathrm{m}^{2}$ and waist circumference $>102 \mathrm{~cm}$ for men and $>88 \mathrm{~cm}$ for women.

\section{Clinical procedures}

Full mouth periodontal clinical parameters were obtained by a single previously trained examiner (F.S.P.), and subsequently calibrated by a goldstandard examiner (J.R.C.). The training and calibration processes followed the method described by Araujo, et al. ${ }^{32}$ (2003) and were performed both prior to the study and before the final exam. An intraexaminer 0.85 correlation coefficient (Kappa test) for probing pocket depth measurements showed high examiner reliability.

Periodontal clinical parameters were obtained prior to therapy (baseline) and at intervals of 3,6 , and 9 months post-therapy. CAL, PD, plaque Index ${ }^{33}$ (PI), and gingival index ${ }^{34}$ (GI) were obtained with a manual periodontal probe (Hu-friedy - Chicago, IL, USA) of all teeth from six periodontal sites.

Initially, participants underwent oral hygiene instructions. Then, as described by Quirynen, et al. $^{35}$ (1995) the one-stage full-mouth disinfection protocol for periodontal therapy was performed by an experienced and trained periodontist (F.S.P).
The protocol consisted of full-mouth periodontal debridement within 24 hours, in two sessions of one hour each, tongue brushing with $1 \%$ chlorhexidine gel for 1 minute, subgingival irrigation with $1 \%$ chlorhexidine gel after scaling, and mouthwashes with $0.12 \%$ chlorhexidine for 30 seconds at the beginning and at the end of each session, with gargling in the final 10 seconds. In addition, during fourteen days, twice-daily, $0.12 \%$ chlorhexidine was used. Every three months, patients underwent oral hygiene instructions, dental prophylaxis and supragingival dental scaling.

\section{Microbiological monitoring}

As described in our previous study, subgingival plaque samples were collected. ${ }^{36}$ Supragingival dental plaque was removed using a sterile curette, and a sterile paper point (fine, Johnson and Johnson, New Brunswick, NJ, USA) was inserted into the gingival sulcus/periodontal pocket and kept there for 10 seconds. Then, paper point was placed in a vial containing $1.0 \mathrm{ml}$ of phosphate-buffered saline (pH7.4), inserted in a minitube and kept on ice. To obtain bacterial dispersion, a vortex mixer at maximum speed was used for $1 \mathrm{~min}$ and stored in a freezer at $-80^{\circ} \mathrm{C}$ until further analyses.

Following the manufacturer's specifications, the genomic DNA (gDNA) was extracted and purified using a commercial Genomic DNA Mini Kit (Life Technologies, Carlsbad, CA, USA).

The total microbial count of Tannerella forsythia, Porphyromonas gingivalis, Treponema denticola, and Aggregatibacter actinomycetemcomitans was performed by quantitative real-time polymerase chain reaction ( $q P C R$ ) using a set of TaqMan (Life Technology, Carlsbad, CA, USA ) primers/probes in Real-Time PCR System, following manufacturer's instructions. The qPCR conditions were: $50^{\circ} \mathrm{C}$ for 2 $\min , 95^{\circ} \mathrm{C}$ for $10 \mathrm{~min}, 40 \mathrm{cycles}$ of $95^{\circ} \mathrm{C}$ for $15 \mathrm{~s}$, and $60^{\circ} \mathrm{C}$ for $1 \mathrm{~min}$. Figure 1 lists the primers and probes used in the study.

\section{Statistical analysis}

Statistical analysis was performed by the GraphPad Prism statistical program (GraphPad Software, La Jolla, CA, USA). First, data were submitted to the Kolmorogov-Smirnov normality test. Demographic data were accessed by chi-square test to verify the group homogeneity. Patient served as unit to evaluate clinical parameters. Intra and inter-group comparisons 


\begin{tabular}{|l|l|}
\hline \multicolumn{1}{|c|}{ Microrganism } & \multicolumn{1}{c|}{ Primers } \\
\hline P. gingivalis & For: ACCTTACCCGGGATTGAATTG \\
\hline & Rev: CAACCATGCAGCACCTACATAGAA \\
\hline & Probe: TGACTGATGGTGAAAACCGTCTTCCCTTC \\
\hline T. forsythia & For: AGCGATGGTAGCAATACCTGTC \\
\hline & Rev: TTCGCCGGGTTATCCCTC \\
\hline & Probe: CACGGGTGAGTAACG \\
\hline T. denticola & For: CCGAATGTGCTCATTTACATAAAGGT \\
\hline & Rev: GATACCCATCGTTGCCTTGGT \\
\hline A. actinomycetemcomitans & Probe: ATGGGCCCGCGTCCCATTAGC \\
\hline & Rev: GCGGCCAAAGTTTTTTCTTTTTCTT \\
\hline & Probe: CCGGATTGGGACTAATT \\
\hline Universal & For: TGGAGCATGTGGTTTAATTCGA \\
\hline & Rev: TGCGGGACTTAACCCAACA \\
\hline & Probe: CACGAGCTGACGACAAGCCATGCA \\
\hline
\end{tabular}

Figure 1- Specific primers and probes used in the study

were assessed by the two-factor repeated-measures ANOVA. Microbial counts were log-transformed, and a Q-Q plot of residual values showed acceptable levels of normality. All analyses were performed with $5 \%$ a.

\section{Results}

For this study, one hundred and four individuals were selected and divided into two groups (obese and non-obese). As ten subjects from the obese group did not attend the baseline appointment, only 94 participants were evaluated. Five individuals of the obese group and two of the non-obese were lost during follow-up visits. Figure 2 shows the flow diagram of the study design.

Table 1 shows participants' sociodemographic and behavioral characteristics, and health conditions for both groups. There was no significant difference between the groups regarding age, sex, ethnicity, diabetes, and smoking habits. Obese individuals showed increased BMI and waist circumference. Moreover, the obese groups had a greater prevalence of participants with arterial hypertension $(P=0.001)$.

All subjects reported no side effect caused by the treatment.

\section{Clinical outcomes}

Table 2 shows periodontal clinical parameters observed in both groups at baseline, 3, 6, and 9 months post-treatment. Periodontal chemical- mechanical therapy using the one-stage full-mouth disinfection protocol significantly improved all clinical parameters (PD, CAL, GI, and PI) for both groups $(p<0.05)$. However, the two of them equally responded to non-surgical periodontal therapy with no statistically significant differences in CAL, PD, GI, and PI after 9 months.

\section{Microbiological results}

Table 3 shows microbial count at baseline, 3, 6, and 9 months. Total bacterial load was not significantly different among follow-up periods and groups $(p<0.05)$. Within 9 months, $P$. gingivalis and A. actinomycetemcomitans significantly decreased in both groups $(p<0.05)$ with no significant difference between groups. At 6 months, T. forsythia decresead significantly in the non-obese group, whereas for the obese group small counts were observed only at 3 months. These reductions, however, were not maintained in both groups at 9 months. Although no difference between groups was observed at any period, periodontal treatment reduced $T$. denticola count within the obese group $(p<0.05)$.

\section{Discussion}

The objective of this 9-month clinical study was to determine whether obese individuals present a worse response to periodontal therapy than non-obese ones. Although few studies have been performed on 
this matter, this study was conducted because (1) the literature presents controversial data, ${ }^{26}(2)$ most studies have 2-6 months follow-ups, ${ }^{22-25}$ (3) studies combining mechanical and chemical procedures are lacking and (4) systematic reviews indicate the need for further studies. ${ }^{26,27}$

The main findings presented here indicate that obesity (BMI $>30 \mathrm{~kg} / \mathrm{m}^{2}$ ) does not affect the clinical and microbiological outcomes of non-surgical periodontal therapy. As obesity has recently become pandemic, ${ }^{37}$ groups were divided into non-obese and obese individuals to achieve a greater reproducibility and reliability for the overall population. Besides, a stratified preliminary analysis showed no significant difference between overweight and normal-weight for all parameters. Thus, as the aim was to assess whether obese people had a worse response to periodontal therapy than non-obese, overweight was included in

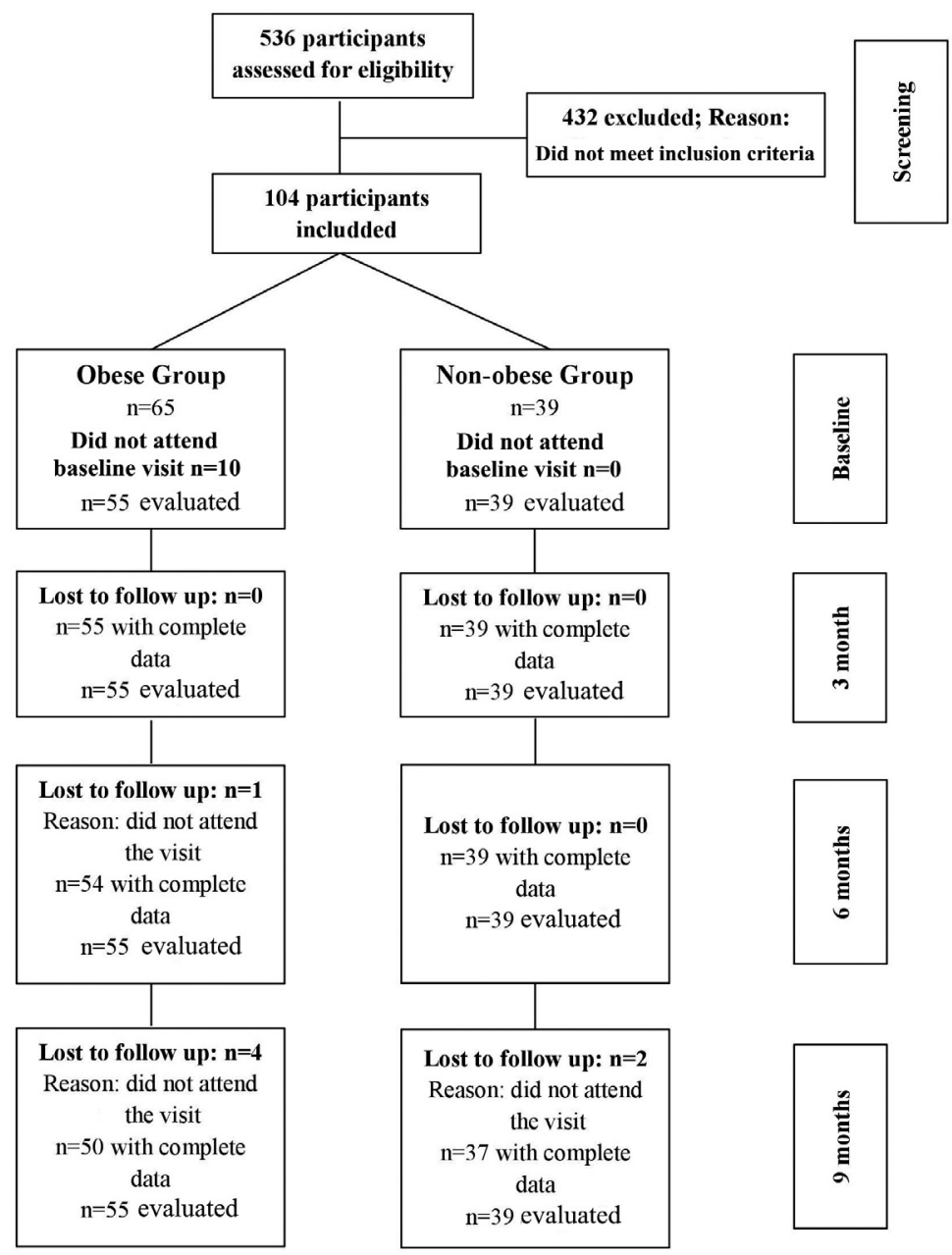

Figure 2- Flow chart of the study design

Table 1- Sociodemographic, behavioral and health condition characteristics of Obese and non-Obese groups

\begin{tabular}{cccc}
\hline Variables & Non-Obese $(\mathbf{n = 3 9 )}$ & Obese $(\mathbf{n = 5 5 )}$ & $\mathbf{p}$-Value \\
\hline Mean age in years (SD) & $50.7(7.1)$ & $48.9(7.8)$ & 0.94 \\
\hline Gender: Male (\%) & $14(35.9)$ & $19(34.5)$ & 0.89 \\
\hline Ethnicity: Non-Caucasian (\%) & $3(7.7)$ & $7(12.7)$ & 0.51 \\
\hline BMI (mean SE) & $25.8(0.5)$ & $36.12(0.57)$ & $0.001^{*}$ \\
\hline Waist circumference (mean SE) & $90.34(1.86)$ & $110.89(1.4)$ & $0.001^{*}$ \\
\hline n (\%) diabetes & $2(5.1)$ & $9(16.4)$ & 0.11 \\
n (\%) of arterial hypertension & $3(7.7)$ & $30(54.5)$ & $0.001^{*}$ \\
Number (\%) of smokers & $5(12.8)$ & $4(7.3)$ & 0.48 \\
\hline
\end{tabular}

*: Indicate significant differences between groups $(p<0.05)$.

$\mathrm{n}$ : number; SE: standard error; SD: standard deviation 
the non-obese group. Although one may speculate that

BMI is a general indicator of body size and not obesity, demographic data demonstrated significant differences in waist circumference between groups, considered a reliable indicator of intra-abdominal adipose tissue and increased risk of cardiometabolic disease. ${ }^{38}$

Table 2- Means ( $\pm S D$ ) of full-mouth clinical parameters for both groups at baseline and follow-up visits

\begin{tabular}{|c|c|c|c|c|}
\hline Variable & Time point & $\begin{array}{c}\text { Non-Obese } \\
n=39\end{array}$ & $\begin{array}{c}\text { Obese } \\
n=55\end{array}$ & p-values \\
\hline \multirow[t]{4}{*}{$\mathrm{PD}(\mathrm{mm})$} & Baseline & $2.98 \pm 0.5$ & $2.90 \pm 0.3$ & 0.553 \\
\hline & 3 months & $2.34 \pm 0.4^{*}$ & $2.26 \pm 0.3^{*}$ & 0.413 \\
\hline & 6 months & $2.22 \pm 0.5^{*}$ & $2.24 \pm 0.4^{*}$ & 0.87 \\
\hline & 9 months & $2.27 \pm 0.5^{*}$ & $2.22 \pm 0.4^{*}$ & 0.97 \\
\hline \multirow[t]{4}{*}{$\mathrm{CAL}(\mathrm{mm})$} & Baseline & $4.23 \pm 1.2$ & $4.03 \pm 0.9$ & 0.525 \\
\hline & 3 months & $3.66 \pm 1.2^{*}$ & $3.61 \pm 1.0^{*}$ & 0.814 \\
\hline & 6 months & $3.64 \pm 1.1^{*}$ & $3.50 \pm 1.0^{*}$ & 0.549 \\
\hline & 9 months & $3.67 \pm 1.1^{*}$ & $3.53 \pm 1.0^{*}$ & 0.705 \\
\hline \multirow[t]{4}{*}{ PI } & Baseline & $0.54 \pm 0.32$ & $0.51 \pm 0.36$ & 0.624 \\
\hline & 3 months & $0.45 \pm 0.31^{*}$ & $0.39 \pm 0.22^{*}$ & 0.25 \\
\hline & 6 months & $0.33 \pm 0.23^{*}$ & $0.37 \pm 0.21^{*}$ & 0.282 \\
\hline & 9 months & $0.32 \pm 0.20^{*}$ & $0.32 \pm 0.22^{*}$ & 0.813 \\
\hline \multirow[t]{4}{*}{$\mathrm{GI}$} & Baseline & $0.32 \pm 0.32$ & $0.37 \pm 0.25$ & 0.431 \\
\hline & 3 months & $0.29 \pm 0.19$ & $0.28 \pm 0.14^{*}$ & 0.662 \\
\hline & 6 months & $0.25 \pm 0.20^{*}$ & $0.24 \pm 0.26^{*}$ & 0.806 \\
\hline & 9 months & $0.27 \pm 0.15^{*}$ & $0.23 \pm 0.22^{*}$ & 0.218 \\
\hline
\end{tabular}

*: indicate significant differences when compared with baseline by repeated measures ANOVA and Tukey's tests $(p<0.05)$.

PD, probing depth; CAL, clinical attachment level; PI: plaque index; GI: gingival index; n: number; SD: standard deviation.

Table 3- Mean number of periodontal pathogens (SE) for both groups at baseline and at follow-up visits

\begin{tabular}{|c|c|c|c|c|}
\hline Bacterial specie & Time point & $\begin{array}{c}\text { Non-Obese } \\
n=39\end{array}$ & $\begin{array}{c}\text { Obese } \\
n=55\end{array}$ & $\begin{array}{r}\text { ANOVA } \\
\text { p-values }\end{array}$ \\
\hline \multirow[t]{4}{*}{$P$. gingivalis } & Baseline & $21.03(8.77)$ & $17.06(4.62)$ & 0.653 \\
\hline & 3 months & $17.07(10.53)$ & $3.65(1.22)^{*}$ & $0.030 \#$ \\
\hline & 6 months & $5.10(2.15)^{\star}$ & $9.81(2.81)^{\star}$ & 0.185 \\
\hline & 9 months & $10.58(4.79)^{*}$ & $13.88(5.49)^{\star}$ & 0.72 \\
\hline \multirow[t]{4}{*}{ T. forsythia } & Baseline & $50.38(21.51)$ & $58.52(18.77)$ & 0.785 \\
\hline & 3 months & $33.31(11.85)$ & $22.05(7.20)^{*}$ & 0.376 \\
\hline & 6 months & $21.36(6.89)^{\star}$ & $55.87(16.24)$ & $0.025 \#$ \\
\hline & 9 months & $45.70(21.50)$ & $33.24(8.79)$ & 0.533 \\
\hline \multirow[t]{4}{*}{ T. denticola } & Baseline & $11.66(4.55)$ & $18.14(6.01)$ & 0.393 \\
\hline & 3 months & $6.52(2.22)$ & $5.64(1.51)^{*}$ & 0.715 \\
\hline & 6 months & $9.60(2.80)$ & $14.77(4.09)$ & 0.279 \\
\hline & 9 months & $9.99(2.53)$ & $7.14(2.32)^{*}$ & 0.482 \\
\hline \multirow[t]{4}{*}{ A. actinomycetemcomitans } & Baseline & 73.36(11.75) & $92.04(20.66)$ & 0.227 \\
\hline & 3 months & $71.57(22.95)$ & $65.55(36.59)$ & 0.877 \\
\hline & 6 months & $46.85(18.09)^{*}$ & $48.22(15.36)^{*}$ & 0.954 \\
\hline & 9 months & $22.28(9.99)^{*}$ & $21.05(6.55)^{*}$ & 0.873 \\
\hline \multirow[t]{4}{*}{ Total load } & Baseline & $11.85(2.41)$ & $12.60(2.41)$ & 0.818 \\
\hline & 3 months & $10.24(1.79)$ & $7.75(1.33)$ & 0.235 \\
\hline & 6 months & $9.12(1.70)$ & $11.22(1.75)$ & 0.39 \\
\hline & 9 months & $8.52(1.44)$ & $9.10(1.61)$ & 0.902 \\
\hline
\end{tabular}

*: indicate significant differences over time by repeated measures ANOVA and Tukey's tests $(p<0.05)$.

\# : Indicate differences between treatment groups at each time point by repeated measures ANOVA and Tukey's tests $(p<0.05)$.

$\mathrm{n}$ : number; SE: standard error 
Periodontal therapy using the one-stage full-mouth disinfection protocol effectively reduced periodontal inflammation, improving all clinical parameters (PD, CAL, and GI). Yet, obese and non-obese individuals showed no significant difference during the study follow-up period. These results might be explained by the disease low severity at baseline, as well as the presence of more smokers within the nonobese group, although not statistically different, Nevertheless, these findings corroborate other clinical studies $^{21,22,39}$ and two systematic reviews, ${ }^{26,27}$ which found that obese and non-obese subjects have a similar periodontal response to nonsurgical mechanical periodontal therapy. Conversely, other studies observed an impaired response to periodontal therapy in obese individuals. ${ }^{23-25}$ These divergent results could be explained by: different follow-up periods, as some studies present 2 months, ${ }^{23} 3$ months ${ }^{21,39}$ and 6 months follow-ups, ${ }^{24,25}$ wheread this study had a 9-month follow-up; differences in sample size and treatment protocol; as well as the inclusion of patients solely with severe periodontitis. ${ }^{25}$

Although clinical studies have shown the efficacy of the one-stage full-mouth disinfection in patients with periodontitis, ${ }^{40}$ this is the first to demonstrate it in obese individuals. The one-stage full-mouth disinfection protocol was chosen for this study for the following reasons: a shorter number of clinical sessions, a shorter treatment time, greater compliance, and cost-effectiveness. ${ }^{35}$

$P$. gingivalis, $T$. forsythia, and T.denticola, usually called "red complex", and A. actinomycetemcomitans are considered the main pathogens associated with periodontal disease. ${ }^{41}$ Some speculate that obese individuals with periodontitis present higher proportions of subgingival periodontal pathogens than normal-weight individuals, increasing the risk for progressive attachment loss and periodontitis development. ${ }^{42}$ In this study, obesity did not impact the changes on overall bacterial load during follow-up. Furthermore, both treatments significantly reduced $P$. gingivalis and $A$. actinomycetemcomitans at 9 months, and $T$. denticola count was only reduced within the obese group. This can be explained by the differences in $T$. denticola count at baseline. As the obese group showed a greater $T$. denticola count, it is reasonable that the periodontal treatment led to a greater reduction within this group. Periodontal therapy did not affect $T$. forsythia count in the obese and non-obese groups. Therefore, there were no major differences in microbial count when comparing obese and non-obese individuals undergoing one-stage full-mouth disinfection. These findings corroborate a recent 3-month prospective clinical study, which found no significant differences in $P$. gingivalis, $T$. forsythia, and $P$. intermedia mean counts between obese and normal-weight individuals on periodontal therapy. ${ }^{43}$

Diabetes is a risk factor for periodontitis. ${ }^{44}$ In this study, two individuals (5.1\%) of the non-obese group and nine $(16.4 \%)$ in the obese group had diabetes. Nonetheless, there was no significant difference between groups, what indicates homogeneity. Moreover, although speculated that patients with diabetes may have a worse periodontal response than non-diabetic, a recent systematic review showed that diabetes mellitus does not significantly impact periodontal clinical parameters of non-surgical periodontal therapy. ${ }^{45}$ In addition, to increase this study external validity, smokers were included. There was no significant difference between groups regarding the number of smokers, but periodontal therapy is proved to be less effective within this population, ${ }^{46}$ which may serve as a possible source of bias. The inclusion of overweight individuals into the control group may have presented a potential bias and a limitation for this study. Furthermore, prospective studies should assess how changes in anthropometric measurements during follow-up may impact results.

Considering the increase of obese individuals, this study results could widely contribute to dental practices. The one-stage full-mouth disinfection seemed a reliable protocol to treat periodontitis in obese individuals.

\section{Conclusion}

Obesity did not affect the clinical and microbiological outcomes of non-surgical periodontal therapy.

\section{Acknowledgments}

The study was financially supported by São Paulo Research Foundation (FAPESP), São Paulo state, Brazil - MSc scholarship (2015/02995-5).

The authors are grateful to PAM Bucarein Joinville Basic Health Unit for their support and help in implementing this project. A special thanks to the nursing team from PAM Bucarein. 


\section{Authors' contributions}

Peralta, Felipe da Silva: Conceptualization (Equal); Data curation (Equal); Formal analysis (Equal); Funding acquisition (Equal); Investigation (Equal); Methodology (Equal); Project administration (Equal); Supervision (Equal); Validation (Equal); Visualization (Equal); Writing-review \& editing (Equal).

Cortelli, Sheila Cavalca: Conceptualization (Lead); Data curation (Equal); Formal analysis (Lead); Funding acquisition (Equal); Investigation (Equal); Methodology (Equal); Project administration (Equal); Resources (Lead); Software (Equal); Supervision (Lead); Validation (Equal); Visualization (Equal); Writing-review \& editing (Lead). Rovai, Emanuel Silva: Data curation (Supporting); Formal analysis (Supporting) Project administration (Supporting); Writing-original draft (Lead); Writing-review \& editing (Lead). Aquino, Davi Romeiro: Conceptualization (Equal); Data curation (Equal); Formal analysis (Equal); Project administration (Equal); Software (Equal); Visualization (Equal); Writing-review \& editing (Equal). Miranda, Taís Browne: Data curation (Equal); Funding acquisition (Equal); Investigation (Equal); Methodology (Lead); Software (Equal); Validation (Equal); Writing-review \& editing (Equal)

Costa, Fernando Oliveira: Conceptualization (Equal); Data curation (Equal); Investigation (Equal); Methodology (Equal); Supervision (Equal); Visualization (Equal); Writing-review \& editing (Lead). Cortelli, José Roberto: Conceptualization (Lead); Formal analysis (Equal); Funding acquisition (Equal); Investigation (Equal); Methodology (Equal); Project administration (Equal); Resources (Equal); Software (Equal); Supervision (Equal); Validation (Equal); Visualization (Equal); Writing-review \& editing (Lead).

\section{References}

1- Eke PI, Wei L, Borgnakke WS, Thornton-Evans G, Zhang X, Lu H, et al. Periodontitis prevalence in adults $\geq 65$ years of age, in the USA. Periodontol 2000. 2016;72(1):76-95. doi: 10.1111/prd.12145

2- Papapanou PN, Sanz M, Buduneli N, Dietrich T, Feres M, Fine DH et al. Periodontitis: consensus report of workgroup 2 of the 2017 World Workshop on the Classification of Periodontal and Peri-Implant Diseases and Conditions. J Periodontol. 2018;89 Suppl 1:S173-82. doi: 10.1002/JPER.17-0721

3- Bartold PM, Van Dyke TE. Host modulation: controlling the inflammation to control the infection. Periodontol 2000. 2017;75(1):317 29. doi: $10.1111 /$ prd.12169
4- Suvan JE, Finer N, D'Aiuto F. Periodontal complications with obesity. Periodontol 2000. 2018;78(1):98-128. doi: 10.1111/prd.12239 5- Foratori-Junior GA, Silva BM, Pinto AC, Honório HM, Groppo FC, Sales-Peres SH. Systemic and periodontal conditions of overweight/ obese patients during pregnancy and after delivery: a prospective cohort. Clin Oral Investig. 2020;24(1):157-65. doi: 10.1007/s00784019-02932-x

6- Barrington G, Khan S, Kent K, Brennan DS, Crocombe LA, Bettiol S. Obesity, dietary sugar and dental caries in Australian adults. Int Dent J. 2019;69(5):383-91. doi: 10.1111/idj.12480

7- Khan S, Bettiol S, Kent K, Barnett T, Peres M, Crocombe LA. Obesity and periodontitis in Australian adults: a population-based crosssectional study. Int Dent J. 2020;70(1):53-61. doi: 10.1111/idj.12514 8- Ylostalo P, Suominen-Taipale L, Reunanen A, Knuuttila M. Association between body weight and periodontal infection. J Clin Periodont. 2008;35(4):297-304. doi: 10.1111/j.1600-051X.2008.01203.x 9- Nascimento GG, Leite FR, Correa MB, Peres MA, Demarco FF. Does periodontal treatment have an effect on clinical and immunological parameters of periodontal disease in obese subjects? A systematic review and meta-analysis. Clin Oral Investig. 2016;20(4):639-47. doi: 10.1007/s00784-015-1678-y

10- Atabay VE, Lutfioğlu M, Avci B, Sakallioglu EE, Aydoğdu A. Obesity and oxidative stress in patients with different periodontal status: a case-control study. J Periodontal Res. 2017;52(1):51-60. doi: 10.1111/ jre. 12368

11- Palle AR, Reddy CM, Shankar BS, Gelli V, Sudhakar J, Reddy KK. Association between obesity and chronic periodontitis: a cross-sectional study. J Contemp Dent Pract. 2013;14(2):168-73. doi: 10.5005/jpjournals-10024-1294

12- Nascimento GG, Peres MA, Mittinty MN, Peres KG, Do LG, Horta BL, et al. Diet-induced overweight and obesity and periodontitis risk: an application of the parametric g-formula in the 1982 Pelotas birth cohort. Am J Epidemiol. 2017;185(6):442-51. doi: 10.1093/aje/kww187 13- Kang J, Smith S, Pavitt S, Wu J. Association between central obesity and tooth loss in the non-obese people: results from the continuous National Health and Nutrition Examination Survey (NHANES) 19992012. J Clin Periodontol. 2019;46(4):430-7. doi: 10.1111/jcpe.13091 14- Gaio EJ, Haas AN, Rösing CK, Oppermann RV, Albandar JM, Susin C. Effect of obesity on periodontal attachment loss progression: a 5-year population-based prospective study. J Clin Periodontol. 2016;43(7):557-65. doi: 10.1111/jcpe.12544

15- Li LW, Wong HM, McGrath CP. Longitudinal association between obesity and periodontal diseases among secondary school students in Hong Kong: a prospective cohort study. BMC Oral Health. 2018;18(1):189. doi: 10.1186/s12903-018-0659-3

16- Chaffee BW, Weston SJ. Association between chronic periodonta disease and obesity: a systematic review and meta-analysis. J Periodontol. 2010;81(12):1708-24. doi: 10.1902/jop.2010.100321 17- Keller A, Rohde JF, Raymond K, Heitmann BL. Association between periodontal disease and overweight and obesity: a systematic review. J Periodontol. 2015;86(6):766-76. doi: 10.1902/jop.2015.140589 18- Martinez-Herrera M, Silvestre-Rangil J, Silvestre FJ. Association between obesity and periodontal disease: a systematic review of epidemiological studies and controlled clinical trials. Med Oral Pato Oral Cir Bucal. 2017;22(6):e708-15. doi: 10.4317/medoral.21786 19- Wilson JA, Clark JJ. Obesity: impediment to wound healing. Crit Care Nurs Q. 2003;26(2):119-32. doi: 10.1097/00002727-20030400000006

20- Doyle SL, Lysaght J, Reynolds JV. Obesity and post-operative complications in patients undergoing non-bariatric surgery. Obes Rev. 2010;11(12):875-86. doi: 10.1111/j.1467-789X.2009.00700.x 21- Zuza EP, Barroso EM, Carrareto AL, Pires JR, Carlos IZ, Theodoro LH, et al. The role of obesity as a modifying factor in patients undergoing non-surgical periodontal therapy. J Periodontol. 2011;82(5):676-82. doi: 10.1902/jop.2010.100545 
22- Al-Zahrani MS, Alghamdi HS. Effect of periodontal treatment on serum C-reactive protein level in obese and normal-weight women affected with chronic periodontitis. Saudi Med J. 2012;33(3):309-14 23- Suvan J, Petrie A, Moles DR, Nibali L, Patel K, Darbar U, et al. Body mass index as a predictive factor of periodontal therapy outcomes. J Dent Res. 2014;93(1):49-54. doi: 10.1177/0022034513511084 24- Gonçalves TE, Feres M, Zimmermann GS, Faveri M, Figueiredo LC, Braga PG, et al. Effects of scaling and root planing on clinical response and serum levels of adipocytokines in patients with obesity and chronic periodontitis. J Periodontol. 2015;86(1):53-61. doi: 10.1902/ jop. 2014.140266

25- Suvan J, Harrington Z, Petrie A, Patel K, Darbar U, Donos N, D'Aiuto F. Obesity as predictive factor of periodontal therapy clinical outcomes: a cohort study. J Clin Periodontol. Forthcoming 2020. doi: $10.1111 /$ jcpe. 13261

26- Papageorgiou SN, Reichert C, Jäger A, Deschner J. Effect of overweight/obesity on response to periodontal treatment: systematic review and a meta-analysis. J Clin Periodontol. 2015;42(3):247-61. doi: $10.1111 /$ jcpe. 12365

27- Akram Z, Safii SH, Vaithilingam RD, Baharuddin NA, Javed F, Vohra F. Efficacy of non-surgical periodontal therapy in the management of chronic periodontitis among obese and non-obese patients: a systematic review and meta-analysis. Clin Oral Investig. 2016;20(5):903-14. doi: 10.1007/s00784-016-1793-4

28- Tonetti MS, Greenwell H, Kornman KS. Staging and grading of periodontitis: Framework and proposal of a new classification and case definition. J Periodontol. 2018;89 Suppl 1:S159-72. doi: 10.1002/ JPER.18-0006

29- Braga RR, Carvalho MA, Bruña-Romero O, Teixeira RE, Costa JE, Mendes EM, et al. Quantification of five putative periodontal pathogens in female patients with and without chronic periodontitis by realtime polymerase chain reaction. Anaerobe. 2010;16(3):234-9. doi: $10.1016 / j$.anaerobe.2010.02.007

30- Santuchi CC, Cortelli JR, Cortelli SC, Cota LO, Fonseca DC, Alencar $\mathrm{CO}$, et al. Scaling and root planing per quadrant versus one-stage fullmouth disinfection: assessment of the impact of chronic periodontitis treatment on quality of life - a clinical randomized, controlled trial. J Periodontol. 2016;87(2):114-23. doi: 10.1902/jop.2015.150105 31- World Health Organization - WHO. Obesity and overweight. Geneva: WHO; 2020 [cited 2020 Mar 10]. Available from: http://www.who.int/ mediacentre/factsheets/fs311/en/

32- Araujo MW, Benedek KM, Benedek JR, Grossi SG, Dorn J, WactawskiWende J, et al. Reproducibility of probing depth measurements using a Constant - force electronic probe: analysis of inter and intraexaminer variability. J Periodontol. 2003;74(12):1736-40. doi: 10.1902/ jop.2003.74.12.1736

33- Silness J, Löe H. Periodontal disease in pregnancy. II. Correlation between oral hygiene and periodontal condition. Acta Odontol Scand. 1964;22:121-35. doi: 10.3109/00016356408993968
34- Löe H, Silness, J. Periodontal disease in pregnancy. I. Prevalence and severity. Acta Odontol Scand. 1963;21:533-51. doi: $10.3109 / 00016356309011240$

35- Quirynen M, Bollen CML, Vandekerckhove BNA, Dekeyser C, Papaioannou W, Eyssen $H$. Full - vs. partial mouth disinfection in the treatment of periodontal infections: short-term clinical and microbiological observations. J Dent Res. 1995;74(8):1459-67. doi: 10.1177/00220345950740080501

36- Cortelli JR, Cortelli SC, Jordan S, Haraszthy VI, Zambon JJ. Prevalence of periodontal pathogens in Brazilians with aggressive or chronic periodontitis. J Clin Periodont. 2005;32(8):860-6. doi: 10.1111/j.1600-051X.2005.00777.x

37- Ford ND, Patel SA, Narayan KM. Obesity in low- and middleincome countries: burden, drivers, and emerging challenges. Annu Rev Public Health. 2017;38:145-64. doi: 10.1146/annurevpublhealth-031816-044604

38- Li R, Shi L, Jia J, Li Y, Yang Q, Ruan Y, et al. Differentiating the associations of waist circumference and body mass index with cardiovascular disease risk in a Chinese population. Asia Pac J Public Health. 2015;27(2):NP457-67. doi: 10.1177/1010539512465306 39- Altay U, Gürgan CA, Ağbaht K. Changes in inflammatory and metabolic parameters after periodontal treatment in patients with and without obesity. J Periodontol. 2013;84(1):13-23. doi: 10.1902/ jop. 2012.110646

40- Lang NP, Tan WC, Krahenmann MA, Zwahlen M. A systematic review of the effects of full-mouth debridement with and without antiseptics in patients with chronic periodontitis. J Clin Periodontol. 2008;35(8 Suppl):8-21. doi: 10.1111/j.1600-051X.2008.01257.x

41- Socransky SS, Haffajee AD, Cugini MA, Smith C, Kent RL Jr. Microbial complexes in subgingival plaque. J Clin Periodontol. 1998;25(2):13444. doi: 10.1111/j.1600-051x.1998.tb02419.x

42- Maciel SS, Feres M, Gonçalves TE, Zimmermann GS, Silva HD, Figueiredo LC, et al. Does obesity influence the subgingival microbiota composition in periodontal health and disease? J Clin Periodontol. 2016;43(12):1003-12. doi: 10.1111/jcpe.12634

43- Tahir KM, Malek AH, Vaithilingam RD, Saub R, Safii SH, Rahman $M T$, et al. Impact of non-surgical periodontal therapy on serum resistin and periodontal pathogen in periodontitis patients with obesity. BMC Oral Health. 2020;20(1):52. doi: 10.1186/s12903-020-1039-3

44- Genco RJ, Borgnakke WS. Risk factors for periodontal disease. Periodontol 2000. 2013;62(1):59-94. doi: 10.1111/j.16000757.2012.00457.x

45- Hsu YT, Nair M, Angelov N, Lalla E, Lee CT. Impact of diabetes on clinical periodontal outcomes following non-surgical periodontal therapy. J Clin Periodontol. 2019;46(2):206-17. doi: 10.1111/ jcpe. 13044

46- Labriola A, Needleman I, Moles DR. Systematic review of the effect of smoking on nonsurgical periodontal therapy. Periodontol 2000. 2005;37:124-37. doi: 10.1111/j.1600-0757.2004.03793.x 